\title{
Production of unique structures using the Aerosol-Through-Plasma (A-T-P) process
}

\author{
C. Luhrs ${ }^{1}$, J. Phillips ${ }^{2}$ \& P. T. Fanson ${ }^{3}$ \\ ${ }^{1}$ Department of Chemical and Nuclear Engineering, \\ University of New Mexico, Albuquerque, USA \\ ${ }^{2}$ Los Alamos National Lab, Los Alamos, USA \\ New Mexico and University of New Mexico National Lab Professor, \\ Department of Mechanical Engineering, Albuquerque, USA \\ ${ }^{3}$ Toyota Technical Center, USA. Inc, Materials Research Department, \\ Ann Arbor, MI 48105, USA
}

\begin{abstract}
Nanoparticle applications for batteries, fuel cells, catalysts and specialty solid fuels, etc, require not simply nanoparticles, but highly engineered nanoparticles. The engineering of chemicals, including particles, requires a thorough, mathematical understanding of processes. Yet, the generation of nano-structures often requires novel processes for material generation that cannot be modeled using existing approaches. In our work we have produced a number of novel nano-structured ceramic structures using an atmospheric pressure microwave through which an aerosol containing precursor species is passed, a technique we call 'Aerosol-Through-Plasma' (A-T-P). For example, ceria-alumina particles of a wide variety of structures, from micron sized hollow spheres to nanoparticles, were produced. In many cases structures are produced that are 'impossible' according to aerosol theory. Examples include the production of nanoparticles from dry, micron scale precursor powders, and the formation of hollow particles from dry precursors. These results and others indicate the need for a new model of particle formation using the A-T-P process. An accurate model can accelerate the engineering of the process to commercial scale.

Keywords: plasma, aerosol, nano, particles, ceria, alumina, metal, synthesis.
\end{abstract}




\section{Introduction}

Almost ten years ago we introduced the Aerosol-Through-Plasma (A-T-P) technique for generating particulate materials. In brief, this technology consists of passing aerosols through microwave generated atmospheric pressure plasma to create particulates, often unique particulates, which can be captured downstream. To date, patented A-T-P technologies from our team include the production of supported metal catalysts $[1,2]$, metal nanoparticles $[3,4]$, micron scale particles [5], ceramic spheres of any size [6,7], spherical boron nitride [8,9] and carbon nanotubes $[10,11]$. There are pending patent applications for oxide nanoparticles, graphite coated metal nanoparticles and multi-cationic particles [12].

In addition to establishing the viability of this new method for producing a wide range of particulates, the empirical work conducted to date suggests that the existing models of particle production from aerosols do not apply to particles produced using the A-T-P method. For example, the existing models for nanoparticle production from aerosols all are based on each primary nanoparticle forming from one liquid drop $[13,14]$. As the liquid evaporates, the dissolved solid precursor precipitates. Hence, according to these models a nanoparticle can be formed during aerosol synthesis from very small (nano) liquid drops containing high concentrations of dissolved precursor, or from larger liquid drops containing very low concentrations of precursor. Yet, in work to date using the A-T-P method a number of different categories of nanoparticles have been formed from precursors containing no liquids. Metal and metal oxide nanoparticles were formed from dry micron-scale metal particles [3,4]. Coreshell nanoparticles were formed from thoroughly dry salts [12].

Below we describe some of the experimental work conducted to demonstrate the need for new models of aerosol synthesis. As the current generation of models are well entrenched in the literature, it is necessary that the experimental work provide unequivocal evidence that there is a need for new models at least for plasma based aerosol systems, but possibly for all methods for aerosol synthesis of particulates.

\section{Experimental}

The apparatus employed in all the work is shown in Figure 1. In each case an aerosol containing gases and/or liquids and selected particle precursors is generated below the plasma, passed through the hot zone of the plasma (temperature $>3500 \mathrm{C}$ ) and then into a chimney where extremely fast cooling takes place. Particles, even nanoparticles, are trapped on filter paper. More elaborate systems employed to trap nanoparticles appear to indicate that simply using filter paper allows more than $90 \%$ of particles to be captured.

In general, both the 'plasma gas' and the 'aerosol gas' (see Figure 1) are pure argon. However, the microwave system, unlike RF systems of similar power levels $(<1200 \mathrm{~W})$, can sustain many different pure (e.g. nitrogen, helium, oxygen) and mixed gas plasmas (e.g. $6 \% \mathrm{H}_{2} / \mathrm{Ar}, \mathrm{O}_{2} / \mathrm{Ar}$ ). This is significant as the 
identity of the gas mixture can influence particle structure. For example, oxide nanoparticles, rather than metal nanoparticles, are formed from larger (ca. 50 micron) dry metal precursors simply by the addition of oxygen (ca. 1\%) to the argon. The addition of small amounts of hydrocarbon to the plasma gas (ca. 1\%) can lead to the formation of carbon/graphite encapsulated metal nanoparticles. Pure nitrogen plasma is required to produce spherical boron nitride particles $[8,9]$, and a mixture of carbon monoxide and argon is used in the production of carbon nanotubes $[10,11]$.

The 'dry' precursor for multi-cationic particles is generated by drying a solvent, either water or ethanol, containing precursor salts (generally nitrates), at $80 \mathrm{C}$.

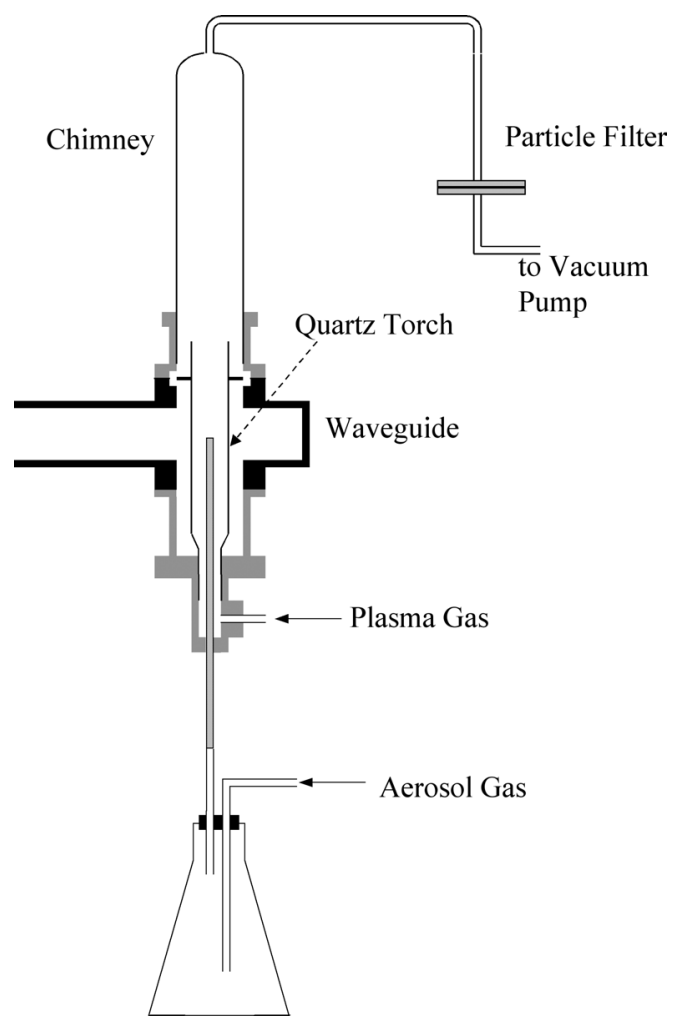

Figure 1: $\quad$ Plasma Torch System. The quartz torch (19 mm OD, approx. 20 $\mathrm{cm}$ tall) can be operated at power as high as $1000 \mathrm{~W}$ for prolonged periods without damage. A variety of aerosol generators has been used. For dry powders generally a small Erlenmeyer flask is employed (ca. $100 \mathrm{cc}$ volume), and the powder agitated simply by directing the aerosol gas, as shown. For liquids (no results reported here), a round bottom flask placed in a sonicator is generally used, and the tubing leading to the plasma is heated to about $100 \mathrm{C}$. 
The resulting precipitate is thoroughly dry. The powders were removed from the beaker by simply scrapping and crushed in an agate mortor. Hence, in this study no liquids were present in the aerosol.

\section{Results and discussion}

Only two different types of particles are presented in this paper in detail: production of aluminum oxide nanoparticles and the formation of hollow particles of various $\mathrm{Ce}$ :Al ratios. These are only two of many examples of particles forming from dry powders with morphologies seemingly in conflict with the expectations of the standard models of particle formation from aerosols. Other examples of dry powder synthesis resulting in 'impossible' morphologies, for example metal nanoparticles $[3,4]$ and nano $\mathrm{CeAl}$ core-shell nano particles [12], are described in prior publications.

Spherical aluminum oxide nanoparticles (Figure 2) were generated from irregularly shaped aluminum flake with an average dimension of 50 microns [15]. Oxide, rather than metal, nanoparticles were generated by adding a small

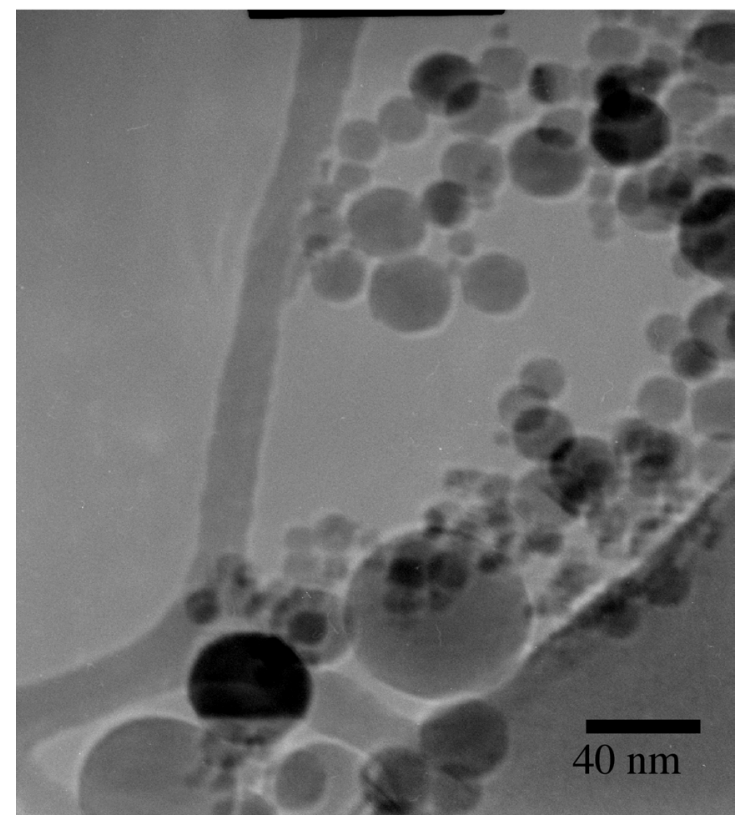

Figure 2: Nano Aluminum Oxide. It can be seen in this TEM image that the average diameter of the particles generated from aluminum flake sent in an $\operatorname{Ar}(>99 \%) / O 2$ aerosol was about $20 \mathrm{~nm}$. (Note: non-spherical objects are lacey carbon grid). 
amount of oxygen $(<1 \%)$ to the argon $(>99 \%)$ aerosol gas. The phase of the oxide particles was influenced by virtually any change in the operating conditions (Figure 3).

The ratio of the final particle volume $(\sim 20 \mathrm{~nm}$ diameter $)$ to that of the initial particle diameter ( $>25$ micron diameter) is about $10^{-9}$. As the final particles also incorporate oxygen, it is clear that each of the initial particles formed more than $10^{9}$ nanoparticles. This could only occur via a process of total vaporization of the initial particles, followed by nucleation of nanoparticles. Clearly, this process is not the one generally employed to model nanoparticle synthesis from standard aerosol processes.

There are a several features of A-T-P system that are fundamentally different from those found in standard thermal aerosol processes. First, the temperature of the neutral gas in an aerosol is far higher than that which can be achieved in a

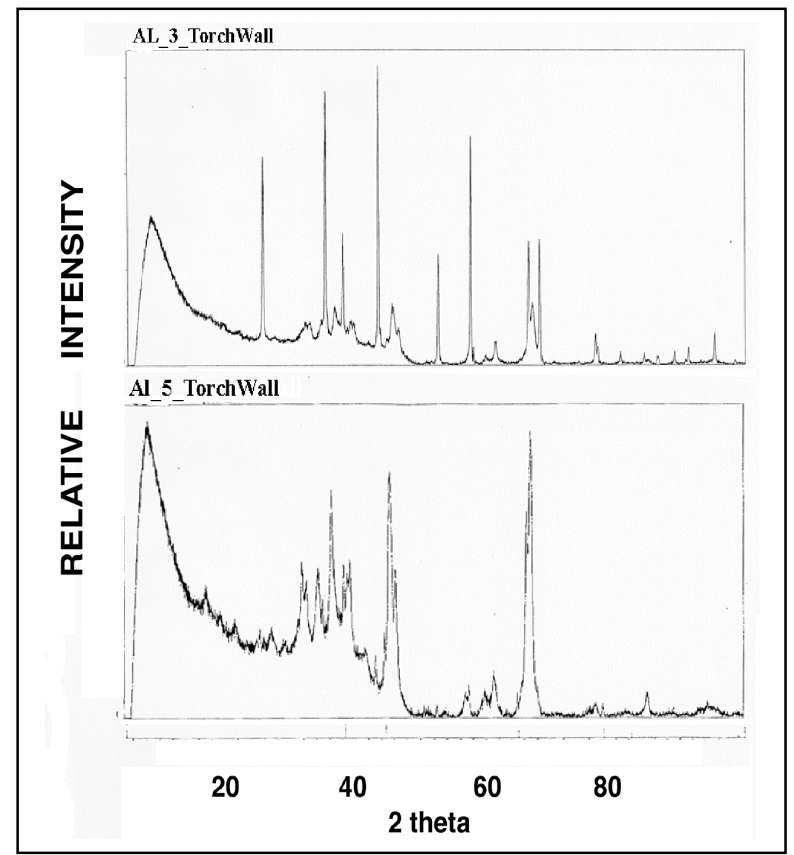

Figure 3: $\quad X R D$ of Nano-Al Oxide. The phase of the particles is a strong function of operating conditions, although the particle sizes were found to be similar. The two samples shown were made with nearly identical conditions, except for power. The top sample was made at a power of $850 \mathrm{~W}$ and is primarily corundum (e.g. four tallest/sharpest reflection lines). The bottom sample was made at $500 \mathrm{~W}$ and is primarily $\delta$ and $\delta$ '. 
thermal oven. As shown in earlier studies the neutral plasma temperature is more than $3500 \mathrm{C}$ even in the afterglow region [16]. Given the rapid cooling this suggests the aerosol particles encounter temperatures greater than $4000 \mathrm{C}$ in the 'hot zone'. Even the best ovens are perhaps able to reach $2500 \mathrm{C}$. Second, in the microwave plasma there are actually two temperatures, that of the neutral species discussed above, and that of the charged species, which in this system has been measured to be as high as $80,000 \mathrm{C}$. As discussed elsewhere, these two 'systems' co-exist in the same space, and exchange energy through 'generation terms' $[17,18]$. Clearly the plasma found in the microwave system is far from equilibrium. Although the fraction of charged species is low, of the order $1 * 10^{-4}$, the huge temperature difference allows for rapid thermal transfer via direct collision and by radiation. Radiant energy exchange is explicitly not included in models of thermal aerosol generation of particles [13]. Third, the rate of cooling is more rapid than in conventional ovens. Even from the wave guide the plasma radiates energy toward the room temperature 'heat sink' surrounding it.

All of the above differences between plasmas and conventional ovens allow metal nanoparticles to be produced from an aerosol containing only micron scale metal particle precursors. The higher temperature of the plasma, as well as the rapid heat transfer via radiation allows the precursor particles to be fully and truly atomized in a very short period of time. A similar model of 'atomization' of solids followed by nucleation is offered to explain (batch) laser processes of nanoparticle production from macroscopic precursors [19]. Also, the more rapid cooling allows the particles to 'freeze' at the nanoscale, probably forming via a 'nucleation' mechanism, before any significant agglomeration growth can occur. In sum, nanoparticle formation from dry macroscopic precursors is one example of the fundamental difference between thermal and plasma production of particles from aerosols.

A second example of unique structure formation from dry powders is hollow particles. The postulated mechanism for hollow particle formation from aerosols is not consistent with the observation of hollow particle formation from dry powders. The existing model is that if the solute exceeds its saturation value during heating, it will begin to precipitate. Given that evaporation occurs from the outside, the first point of precipitation, and the point of greatest supersaturation, will be at the outside of the particle. A hollow shell of precipitate will form via this process. Yet, as shown in Figure 4, and elsewhere [12], the A-T-P process produces hollow particles in the absence of any liquid. Moreover, as shown in Figure 4, there is segregation within the surface itself. Models of hollow particle formation from wet aerosols suggest segregation should occur as a function of distance from the surface. The solute that supersatures first will be on the surface, and those that remain in solution to higher concentrations will be found further below the surface. Indeed, although there are many examples of metal segregation to the surface of hollow oxide particles formed from aerosols, we have been unable to locate any structure equivalent to that shown in Figure 4. We believe the formation of segregated zones of two different oxides in the surface of a single hollow particle is truly unprecedented. 

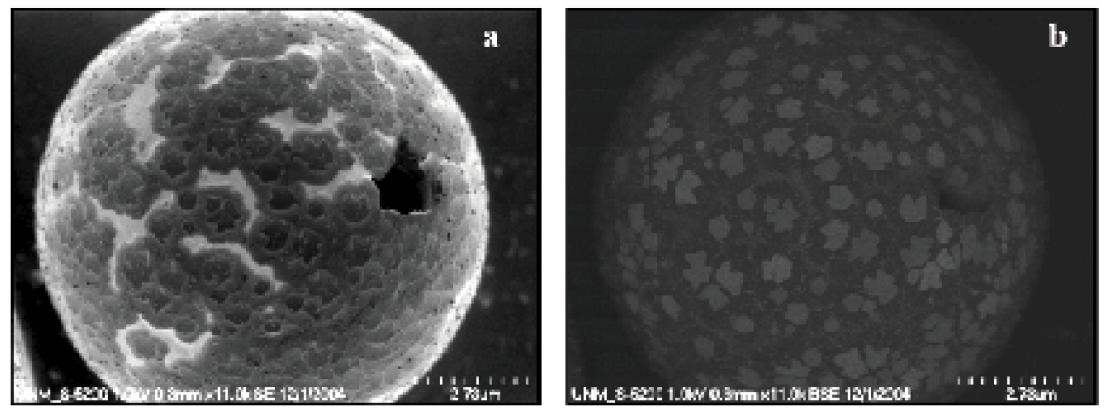

Figure 4: Hollow Ce:Al Particle with Segregation- Particle made from 75 $\mathrm{Ce} / 25 \mathrm{Al}$ evaporated until dry precusor. On the order of $10 \%$ of particles of this composition show surface segregation. A) Secondary electron image from the scanning electron microscope. B) Backscatter image in which the light zones are ceria rich. The cerium rich zones correspond not simply to the light or dark areas on the backscatter image, but specifically to the darker areas ('outlined') in the dark part of the secondary electron image. The particle is clearly hollow.

\section{Summary}

In this article two types of particles produced from an aerosols of dry powders are presented for the first time: nano-oxide particles (alumina) and hollow Ce:Al particles with distinct surface segregation. The existence of both types of particles formed from fully dry aerosols shows that the A-T-P method is not simply a new form of aerosol synthesis. According to standard aerosol theory, the formation of both nano particles and hollow particles requires a liquid drop based process. The extremely high temperatures encountered in a plasma, and particularly the existence of very hot electrons that heat via radiation, are postulated to be the root differences between A-T-P and standard thermal plasmas.

\section{References}

[1] J. Phillips, S. Shim, I. M. Fonseca and S. Carabineiro, 'Plasma Generation of Supported Metal Catalysts', Applied Catalysis 237,41 (2002).

[2] J. Phillips, 'Plasma Generation of Supported Metal Catalysts', U.S. Patent 5,989,648 (1999).

[3] J. Phillips, W. Kroenke and W. L. Perry, 'Method for Producing Metallic Nanoparticles', U. S. Patent 6,689,192 (2004). 
[4] J. C. Weigle, C. C. Luhrs, C. K. Chen, W. L. Perry, J. T. Mang, G. P. Lopez, J. Phillips 'Generation of Aluminum Nanoparticles Using an Atmospheric Pressure Plasma Torch', J. Phys. Chem.B 108,1860. (2004).

[5] J. Phillips, W. Kroenke and W. L. Perry, 'Method for Producing Metallic Microparticles', U.S. Patent 6,755,886. (2004).

[6] J. Phillips, S. Gleiman and C.-K. Chen, 'Method for Producing Ceramic Particles and Agglomerates,' U.S. Patent 6,261,484 (2001).

[7] J. Phillips, S. Gleiman and C.K. Chen, 'Low-power Plasma Torch Method for the Production of Crystalline Spherical Ceramic Particles', J. of Mat. Res. 16,1256 (2001).

[8] S. Gleiman, C.-K. Chen, A. Datye, and J. Phillips, 'Melting and Spheroidization of Hexagonal Boron Nitride in a Microwave-Powered, Atmospheric Pressure Nitrogen Plasma', Journal of Materials Science, 37 (16), 3429-3440. (2002).

[9] J. Phillips, S. Gleiman and C.K. Chen. 'Method For Preparing Spherical Particles Of Boron Nitride', U. S. Patent 6,652,822 (2003).

[10] J. Phillips, W. L. Perry and C.-K Chen, 'Plasma Method for Producing Carbon Nanotubes', U.S. Patent 6,998,103 (2006).

[11] Chun-Ku Chen, William L. Perry and Jonathan Phillips, 'Plasma Torch Production of Macroscopic Carbon Nanotube Structures', Carbon 41, 2555 (2003).

[12] C.C. Luhrs, J. Phillips and P. Fanson, 'Production of Complex CeriumAluminum Oxides Using and Atmospheric Pressure Plasma Torch' Langmuir, in press (published on web).

[13] G. L. Messing, S.C. Zhang, and G.V. Jayanthi, 'Ceramic Powder Synthesis by Spray Pyrolysis’, J. Am. Cer. Soc. 76, 2707 (1993).

[14] A. Gurav, T. Kodas, T. Pluym, Y. Xiong, Aerosol Sci. and Tech. 19, 411 (1993).

[15] J. Phillips, M. Mendoza and C-K. Chen, 'Method of Producing Metal Oxide Nanoparticles' US Patent Application 2004000918 (2004).

[16] C-K. Chen and J. Phillips, 'Impact of Aerosol Particles on the Structure of an Atmospheric Pressure Microwave Plasma Afterglow', J. Phys. D: Applied Physics, 35. 998-1009 (2002).

[17] C.K. Chen, T-C Wei, L. R. Collins and J. Phillips, 'Modeling the Discharge Region of a Microwave Generated Hydrogen Plasma.' J. Phys. D: Appl. Phys., 32(6):688-698 (1999).

[18] Wei, T.-C., L. R. Collins and J. Phillips, 'Measurement and Modeling of Natom Behavior in the Afterglow of a Microwave Plasma', AIChE J., 42(5):1361-1370 (1996).

[19] H. Ferkel, J. Naser and W. Riehemann, 'Laser-induced Solid Solution of the Binary Nanoparticle System A12O3-ZrO2', Nanostructured Materials 8,47 (1997). 